\title{
ARTICLE OPEN Orbitally driven giant thermal conductance associated with abnormal strain dependence in hydrogenated graphene-like borophene
}

\author{
Jia He${ }^{1}$, Dengfeng $\mathrm{Li}^{1}$, Yan Ying ${ }^{1}$, Chunbao Feng ${ }^{1}$, Junjie He $\mathbb{D}^{1}$, Chengyong Zhong $\mathbb{D}^{1}$, Hangbo Zhou ${ }^{2}$, Ping Zhou ${ }^{1}$ and Gang Zhang ${ }^{2}$
}

Heat energy in solids is carried by phonons and electrons. However, in most two-dimensional (2D) materials, the contribution from electrons to total thermal conduction is much lower than that for phonons. In this work, through first-principles calculations combined with non-equilibrium Green's function theory, we studied electron and phonon thermal conductance in recently synthesized 2D hydrogen boride. The hexagonal boron network with bridging hydrogen atoms is suggested to exhibit comparable lattice thermal conductance $\left(4.07 \mathrm{nWK}^{-1} \mathrm{~nm}^{-2}\right)$ as graphene $\left(4.1 \mathrm{nWK}^{-1} \mathrm{~nm}^{-2}\right)$, and similar electron thermal conductance $\left(3.6 \mathrm{nWK}^{-1} \mathrm{~nm}^{-2}\right)$, which is almost ten times that of graphene. As a result, total thermal conductance of 2D hydrogen boride is about two-fold of graphene, being the highest value in all known 2D materials. Moreover, tensile strain along the armchair direction leads to an increase in carrier density, significantly increasing electron thermal conductance. The increase in electron thermal conductance offsets the reduction in phonon thermal conductance, contributing to an abnormal increase in thermal conductance. We demonstrate that the high electron density governs extraordinarily high thermal conductance in 2D hydrogen boride, distinctive among 2D materials.

npj Computational Materials (2019)5:47; https://doi.org/10.1038/s41524-019-0183-2

\section{INTRODUCTION}

Research on atomically thin two-dimensional (2D) materials such as graphene, transition metal dichalcogenides (TMDCs), hexagonal boron nitride ( $\mathrm{h}-\mathrm{BN})$, and black phosphorene is actively pursued due to their remarkable intrinsic properties with great interest in fundamental science and engineering applications. ${ }^{1}$ They offer great opportunities for a variety of applications, including nanoelectronics, optoelectronics, renewable energy and heat dissipation, which are highly expected for realization of atomically thin devices in the near future. ${ }^{2-6}$ With the quickly increased operation speed, and higher integration density, the power consumption in integrated nanoscale devices rapidly climb, which will cause degradation in device performance. In particular, for 2D materials based devices, owing to the atomic thickness, the localized heating in the confined volume is becoming more crucial issue. ${ }^{7,8}$ Moreover, understanding of thermal properties also directly relate to important application of $2 \mathrm{D}$ materials, including the developments of thermal interface materials, ${ }^{9,10}$ thermoelectric energy conversion, ${ }^{11}$ and thermal coating materials. ${ }^{12}$ Obviously, it is indispensable to understand the fundamental principles, and then improve the ability for manipulating thermal properties of $2 \mathrm{D}$ materials, which topic has attracted tremendous attention in recent years. ${ }^{13-16}$

In solid, the heat energy is not only carried by phonon, but also electron, because electrons likewise flow in the presence of a temperature gradient. However, in 2D materials, the contribution of these two heat carriers to total thermal conduction is not analogous. ${ }^{17-21}$ In 2D semiconductor such as $\mathrm{MoS}_{2}$ and black phosphorene, phonon dominates heat conduction. ${ }^{17-19}$ Even though group-IV 2D materials represented by graphene are gapless semi-metal hosting massless Dirac fermions makes them attractive, the low carrier concentration at Fermi level limits the electrical thermal conductance. Stanene, a monolayer of tin atoms, is reported to exhibit comparable electrical thermal conductance at room temperature with respect to the phonon counterpart. ${ }^{22}$ However, its lattice (electron) thermal conductance is only around $0.4 \mathrm{nWK}^{-1} \mathrm{~nm}^{-2}$, which is only $10 \%$ of that of graphene $\left(4.1 \mathrm{nWK}^{-1} \mathrm{~nm}^{-2}\right) .^{23-26}$ Therefore, some important and fundamental questions remain unsolved, for instance, is there a 2D material which exhibits both high electrical and phonon thermal conductance? Is it possible to achieve higher thermal conductance over graphene?

In this work, we demonstrated the coexistence of high phonon and electron thermal conductance in the recently experimentally fabricated 2D hydrogen boride sheet, ${ }^{27}$ via a systematic study of electron and phonon quantum transport using first-principles calculation combined with non-equilibrium Green's function theory. The computational details are shown in Methods section and supporting information, Fig. S1. Unlike the usually observed reduction in phonon thermal conductance induced by tensile strain, the orbitally driven electrical thermal conductance exhibits abnormal strain dependence. As carbon's neighbor in the periodic table, boron has been expected to form graphene-like 2D structure borophene for many years. ${ }^{28-32} 2 \mathrm{D}$ boron sheets, also called borophene, have been successfully synthesized on a $\mathrm{Ag}$ (111) surface. ${ }^{33,34}$ Following the realization of the buckled phase and the planar phase of borophene, extensive theoretical studies

\footnotetext{
${ }^{1}$ School of Science, Chongqing University of Posts and Telecommunications, Chongqing 400065, China and ${ }^{2}$ Institute of High Performance Computing, A*STAR, Singapore 138632, Singapore

Correspondence: Dengfeng Li (lidf@cqupt.edu.cn) or Gang Zhang (zhangg@ihpc.a-star.edu.sg)
}

Received: 22 November 2018 Accepted: 15 March 2019

Published online: 10 April 2019 

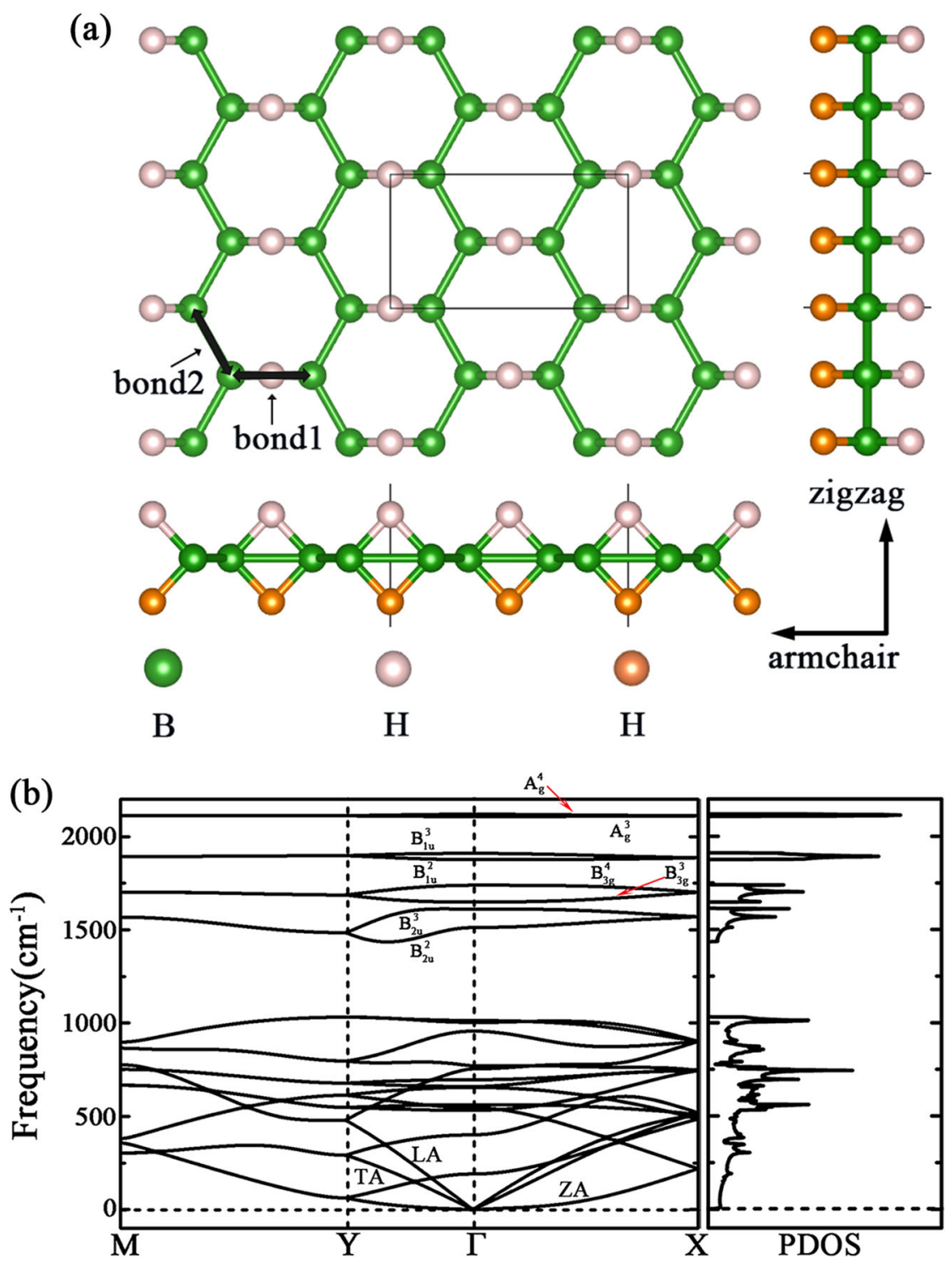

Fig. 1 a The crystal structure of 2D hydrogen boride. The solid lines show the unit cell. $\mathbf{b}$ Phonon dispersion and density of states of $2 \mathrm{D}$ hydrogen boride

were conducted to explore the various applications of borophene such as hydrogen storage, rechargeable metal-ion batteries, superconductors, heat channel and mechanically robust components. ${ }^{35-47}$ In particular, the strain effects on the electronic, optical, and transport properties of borophene are explored. ${ }^{39,44,48-50}$ The fascinating behaviors revealed here in 2D hydrogen boride sheet not only widen our knowledge in thermal transport in 2D materials, but also provide a new route to manipulate the thermal conductance since it is much easier for controlling electrons than controlling phonons.

\section{RESULTS AND DISCUSSION}

Ideal strength and phonon instability

The hydrogenated graphene-like borophene, in another word, 2D hydrogen boride, is illustrated in Fig. 1a. Its unit cell consists of 4 hydrogen atoms and 4 boron atoms. It shows a graphene-like honeycomb lattice with a flat plane made from boron atoms, with two layers of hydrogen atoms are decorated on both sides of the B-B bonds. The optimized lattice constants are $a=5.298 \AA$ and $b=2.987 \AA$. For convenience, we define the B-B bonds bridged by the hydrogen atoms as Bond-1, and the B-B bonds without hydrogen bridging as Bond-2. The lengths of Bond-1 $\left(r_{1}\right)$ and Bond$2\left(r_{2}\right)$ are $1.82 \AA$ and $1.72 \AA$, respectively. The $\mathrm{B}-\mathrm{H}$ bond length was found to be $1.32 \AA$. The structural parameters are in excellent agreement with previous report. ${ }^{51}$

The calculated phonon dispersion is shown in Fig. $1 \mathrm{~b}$. Correspondingly, we present atomic motions of vibrational modes in Fig. S2. There is no sign of imaginary frequencies in the phonon dispersion which accordingly confirms the thermodynamic stability of this attractive 2D material. Around the $\Gamma$ point, there are three acoustic modes, two of them (longitudinal acoustic and transverse acoustic modes, LA and TA) have linear dispersion, and the third one (out-of-plane acoustic mode, ZA) has parabolic dispersion. The high-frequency optical modes $\left(>1500 \mathrm{~cm}^{-1}\right)$ are contributed by $\mathrm{B}-\mathrm{H}$ bonds, and the rest low-frequency phonon modes $\left(\omega<1000 \mathrm{~cm}^{-1}\right)$ are contributed by $B$ atoms, which are lower than those in graphene $\left(\sim 1600 \mathrm{~cm}^{-1}\right)$. As illustrated in Fig. $\mathrm{S} 2$, in high-frequency region, atomic motions associated with $\mathrm{B}_{3 g^{\prime}}^{4}$ $\mathrm{B}_{3 \mathrm{~g}^{\prime}}^{3} \mathrm{~B}_{2 \mathrm{u}^{\prime}}^{3} \mathrm{~B}_{2 \mathrm{u}^{\prime}}^{2}$ occur mostly along the armchair-direction. On the other hand, atomic motions associated with $\mathrm{B}_{3 \mathrm{u}}^{3}, \mathrm{~B}_{1 \mathrm{~g}}^{2}$ occur mostly along the zigzag-direction. 

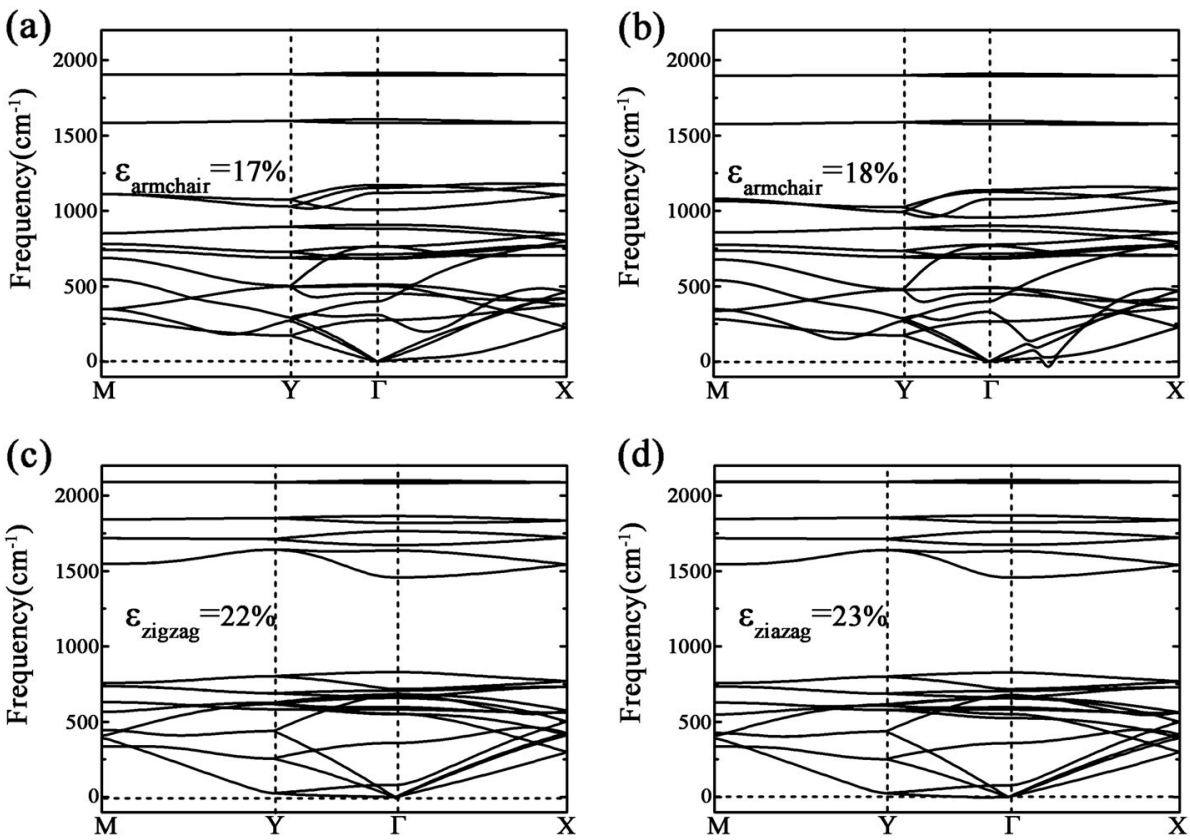

Fig. 2 The phonon dispersion of $2 \mathrm{D}$ hydrogen boride under uniaxial strain along armchair- and zigzag-direction. $\mathbf{a} \varepsilon_{\text {armchair }}=17 \%, \mathbf{b} \varepsilon_{\text {armchair }}=$ $18 \%$, c $\varepsilon_{\text {zigzag }}=22 \%$, d $\varepsilon_{\text {zigzag }}=23 \%$

Strain effect on electronic, optoelectronic, thermal, and thermoelectric properties of $2 \mathrm{D}$ materials has gained tremendous attention. ${ }^{52-55}$ Furthermore, strain dependent phonon dispersion provides direct criteria for the thermodynamic stability of layered materials. Next we explore the phonon dispersion of 2D hydrogen boride under uniaxial tensile and compressive strain. The strain is applied on the rectangular unit cell along the armchair- (or zigzag-) direction, and the lattice is optimized along the lateral direction. The strain is defined as $\varepsilon_{i}=\left(L_{i}-L_{i 0}\right) / L_{i 0}, i=$ armchair/zigzag, where $L_{i}$ is the strained lattice constant along armchair- or zigzagdirection, and $L_{i 0}$ is the corresponding original lattice constant. When $2 \%$ uniaxial compressive strain is applied in armchair or zigzag direction, the structure becomes unstable (shown in Fig. S3). Therefore, in the following, we focus on the effect of tensile strain on the thermal transport of 2D hydrogen boride. Figure 2 shows the phonon dispersions of 2D hydrogen boride under the tensile strain along armchair- and zigzag-direction, respectively. For convenience, the negative $y$-axis is used to show the imaginary frequencies. The phonon mode exhibiting imaginary frequency will grow in amplitude until the structure shifts to a new stable state, demonstrating the instability of crystallographic nature. Under the uniaxial strain in the armchair-direction, softening of phonon modes occurring gradually, and ZA mode along the $\Gamma-X$ direction softened and the frequencies become imaginary when the strain reaches a critical value (between $\varepsilon_{\text {armchair }}=17 \%$ and $\varepsilon_{\text {armchair }}=18 \%$ ). For zigzag-direction strain, one phonon mode becomes unstable firstly with around $23 \%$ tensile strain. The unstable mode is also the out-of-plane ZA mode, indicating 2D hydrogen boride is unstable with respect to long wavelength distortion and is thus elastically unstable under large tensile deformation.

We also studied the ideal strength of 2D hydrogen boride using strain-stress curves under various strains, as shown in Fig. S4. When the strain is applied in the armchair-direction, the stress increases with increasing strain until $\varepsilon_{\text {armchair }}=22 \%$, beyond which the stress decreases sharply. In the zigzag-direction, the ultimate tensile strain is $\varepsilon_{\text {zigzag }}=34 \%$. The corresponding ideal strengths are 19.66 and $17.77 \mathrm{~N} / \mathrm{m}$, respectively. In each case the phonon instability occurs before the peak of the stress-strain curve, indicating the ideal strength of $2 D$ hydrogen boride is dictated by instabilities in the acoustic phonon spectra, which is similar to the case for pristine borophene and hydrogenated borophene. ${ }^{56}$ From the optimized lattice constants along transverse and longitudinal directions, we obtained the Poisson's ratio of $2 \mathrm{D}$ hydrogen boride as 0.26 (armchair direction) and 0.21 (zigzag direction), which are in good agreement with previous report $\left(0.25\right.$ and 0.19 , respectively). ${ }^{44}$

Phonon thermal conductance of 2D hydrogen boride

Next, we employ the phonon NEGF approach to investigate the lattice thermal conductance of 2D hydrogen boride. The scaled thermal conductance is introduced to characterize the thermal transport properties. Herein, the cross-sectional area $S$ is defined as $S=W \times d$, where $W$ is the width and $d$ is the thickness. For 2D hydrogen boride, its thickness is $3.49 \AA$, by adding the diameter of hydrogen atom $(1.60 \AA)$ with the distance of two hydrogen layers, which is $1.89 \AA \AA^{27}$ Figure 3 a shows the scaled thermal conductance of 2D hydrogen boride as a function of temperature along both armchair- and zigzag-direction. The increasing trend of thermal conductance with temperature is a common phenomenon for ballistic phonon transport. ${ }^{24}$ It can be noticed that the phonon thermal conductance at room temperature along armchair- and zigzag-direction are $3.93 \mathrm{nW} \mathrm{K}^{-1} \mathrm{~nm}^{-2}$ and $4.07 \mathrm{nW} \mathrm{K}^{-1} \mathrm{~nm}^{-2}$, which is slightly smaller than that of graphene $\left(\sim 4.1 \mathrm{nW} \mathrm{K}^{-1}\right.$ $\left.\mathrm{nm}^{-2}\right),{ }^{24}$ but much higher than most 2D materials. In 2D hydrogen boride, the effective atomic mass ( $\mathrm{B}-\mathrm{H}$ group) can be considered as same as that of graphene, and the strength of B-B bond and $\mathrm{C}-\mathrm{C}$ bond are comparable. Furthermore, the honeycomb lattice of $2 \mathrm{D}$ hydrogen boride is much similar to that of graphene. As consequence, $2 \mathrm{D}$ hydrogen boride and graphene have comparable lattice thermal conductance.

Figure 4 shows the room temperature phonon thermal conductance $\left(\boldsymbol{\sigma}_{p \text {-armchair }}\right.$ and $\left.\boldsymbol{\sigma}_{p \text {-zigzag }}\right)$ as a function of tensile strain along armchair- and zigzag-direction, respectively. It is clear that armchair-strain leads to quick decrease in $\boldsymbol{\sigma}_{p \text {-armchair }}$ and $\boldsymbol{\sigma}_{p \text { - }}$ zigzag within the whole range of considered strain. On the other

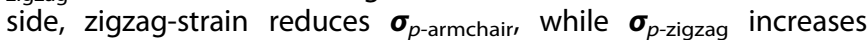
slightly when strain is less than $14 \%$, and then decreases when $\varepsilon_{\text {zigzag }}>16 \%$. Overall, tensile strain results in reduction in lattice 

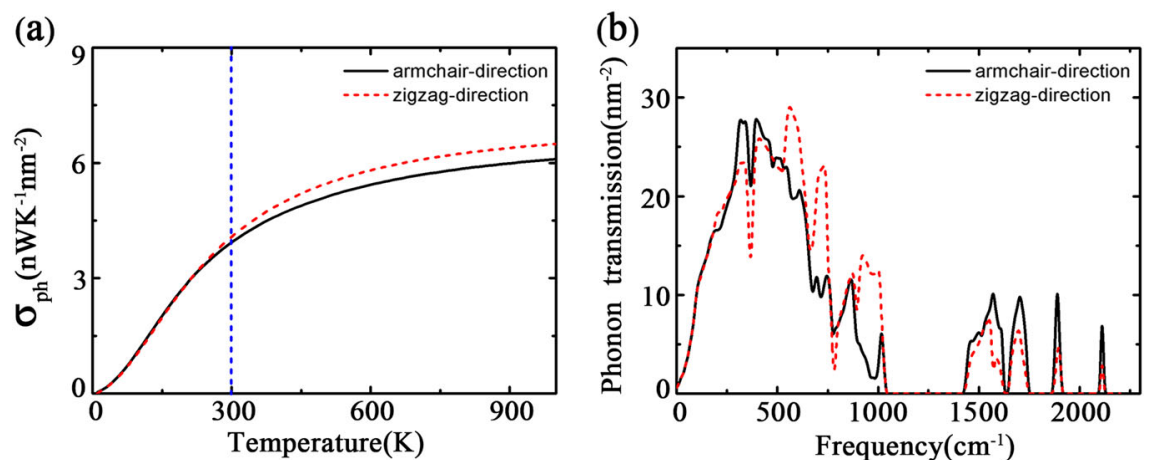

Fig. 3 a Phonon thermal conductance of $2 D$ hydrogen boride versus temperature. b Phonon transmission coefficient $\pi \omega]$ per unit area against phonon frequency for 2D hydrogen boride

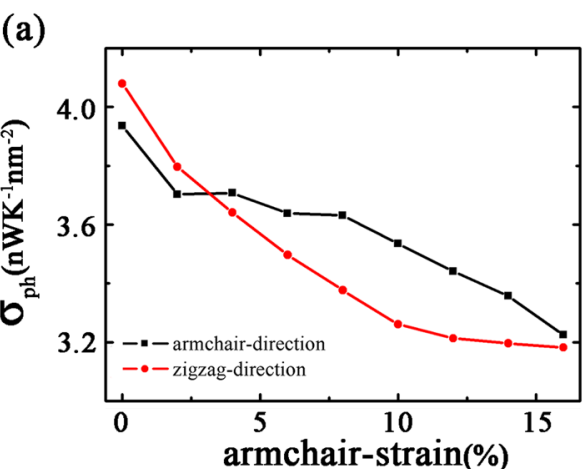

(c)

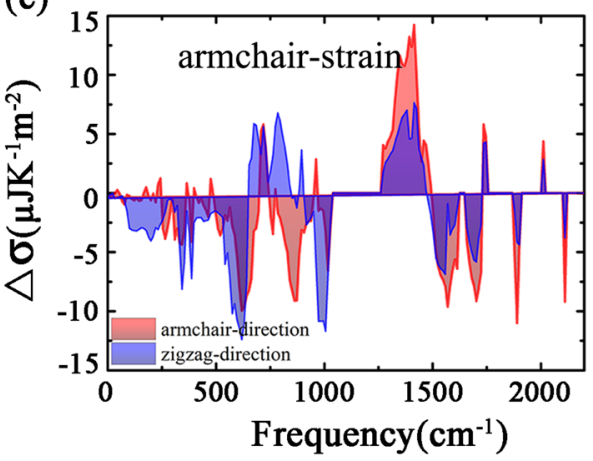

(b)

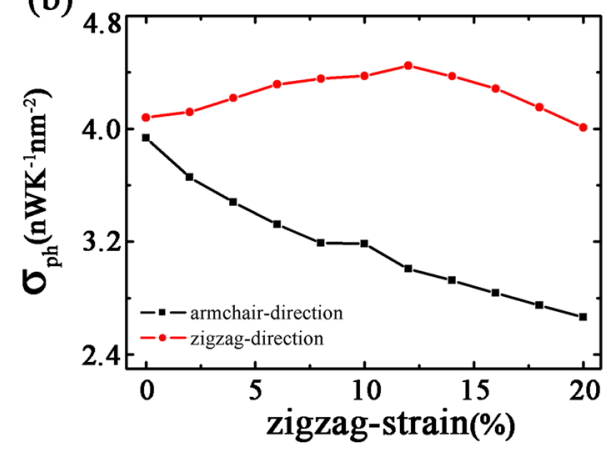

(d)

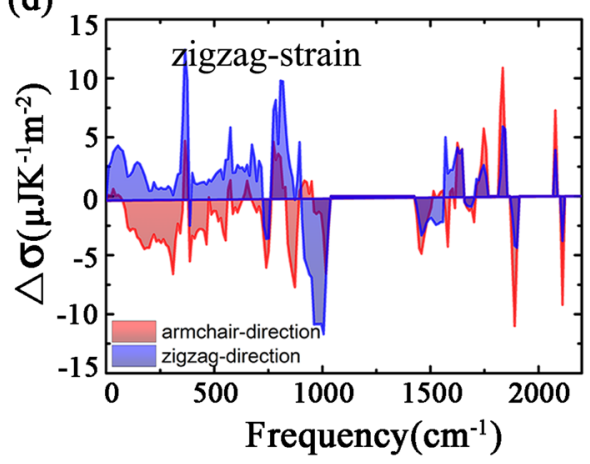

Fig. 4 The room temperature phonon thermal conductance as a function of tensile strain. a Armchair-direction strain and $\mathbf{b}$ zigzag-direction strain. c The differential thermal conductance $\Delta \boldsymbol{\sigma}$ under $8 \%$ armchair-strain. $\mathbf{d}$ The differential thermal conductance $\Delta \boldsymbol{\sigma}$ under $8 \%$ zigzag-strain

thermal conductance, excluding $\boldsymbol{\sigma}_{p \text {-zigzag }}$ which exhibits weak dependence to strain along zigzag-direction.

To understand the nontrivial strain effect on thermal conductance, we present the phonon transmission along the armchair- or zigzag-direction, as shown in Fig. 3b and Fig. S5. When the strain is applied in the armchair-direction, tensile strain results in dispersive phonon modes converged to the lowfrequency region. As shown in Fig. S5(a). and (b), the transmission coefficient in the frequency range of $100-1000 \mathrm{~cm}^{-1}$ decreases, and there has a significant red-shift in the maximum frequency $\left(\omega_{\max }\right)$ in transmission coefficient along armchair- and zigzagdirection. This is consistent with the shifting down in acoustic modes as shown in Fig. 2. All these features result in reduction of thermal conductance upon armchair-strain. On the other side, for zigzag-direction strain, there is also an obvious decrease in the transmission coefficient of armchair-direction, combined with redshift in the high-frequency phonon $\left(750 \sim 1000 \mathrm{~cm}^{-1}\right)$ transmission. For phonon transmission along zigzag-direction, aside to these negative influences, there is slight increase in phonon transmission at the zone center $\left(\omega<100 \mathrm{~cm}^{-1}\right)$, leading to the weak zigzag-strain dependence observed in thermal conductance along zigzag-direction (Fig. 4b).

To further understand how strain affects the relative contribution from phonons at different frequencies, next we investigate the differential thermal conductance, which is defined as

$\Delta \boldsymbol{\sigma}(\omega)=\frac{\hbar \omega}{2 \pi S} \frac{\partial f(\omega)}{\partial T} \Delta \equiv(\omega)$,

where $\Delta \Xi(\omega)$ is the difference in phonon transmission at 8 and $0 \%$ strain. Here we focus on the case at room temperature $300 \mathrm{~K} . \Delta \boldsymbol{\sigma}$ $(\omega)$ identifies the contribution to the change in thermal conductance by phonon mode with frequency $\omega$, with respect to the $8 \%$ tensile strain (armchair- or zigzag-strain).

With strain in the armchair-direction (Fig. 4c), the reduced thermal conductance $\left(\Delta \boldsymbol{\sigma}_{p \text {-armchair }}\right.$ and $\Delta \boldsymbol{\sigma}_{p \text {-zigzag }}$ ) is due to the drop in the phonon transmission in low-frequency regime. 
(a)

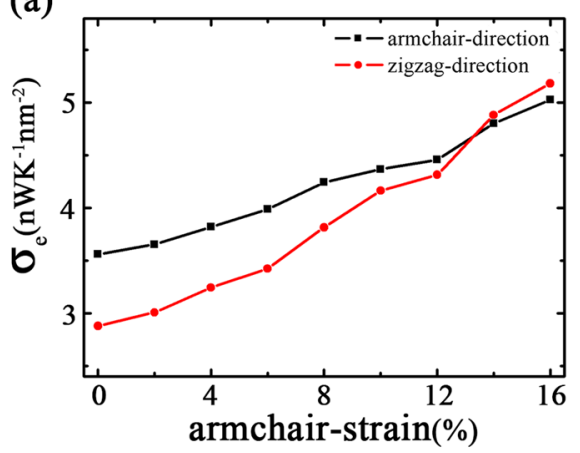

(c)

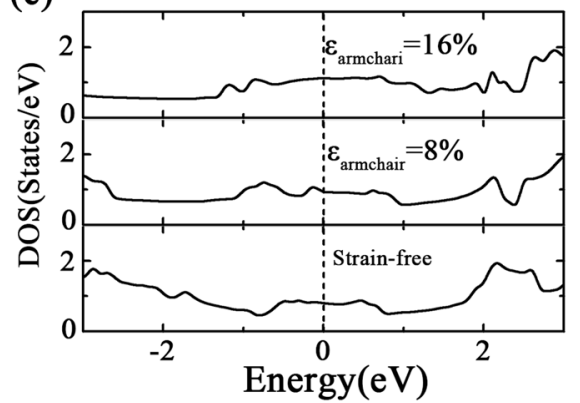

(b)

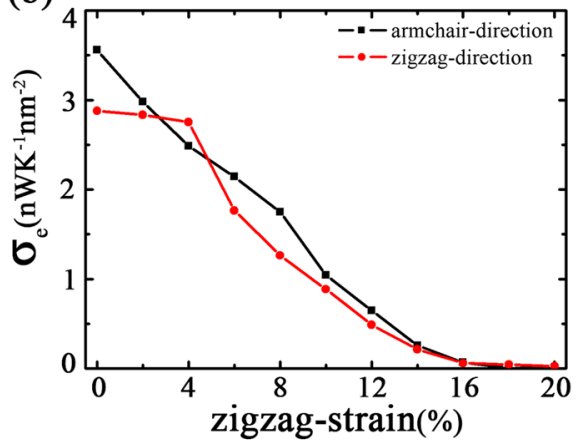

(d)

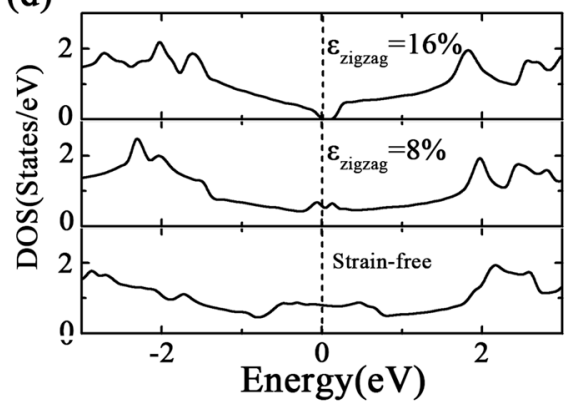

Fig. 5 The room temperature electrical thermal conductance as a function of strain for $\mathbf{a}$ armchair-direction strain and $\mathbf{b}$ zigzag-direction strain. The DOS of 2D hydrogen boride as a function of strain for $\mathbf{c}$ armchair-direction strain and $\mathbf{d}$ zigzag-direction strain

However, when tensile strain is applied in the zigzag-direction

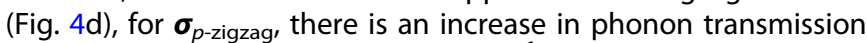
in the frequency range of $0-900 \mathrm{~cm}^{-1}$. On the other side, for $\boldsymbol{\sigma}_{p \text {-armchair, }}$ there is obvious drop over the same frequency range. Therefore, $\boldsymbol{\sigma}_{p \text {-zigzag }}$ increases with zigzag-direction strain, while

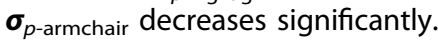

\section{Giant electrical thermal conductance}

Figure S6 (a) illustrates the band structure of strain-free 2D hydrogen boride. It shows that this $2 \mathrm{D}$ material yields metallic character and our predicted band structure matches excellently with the previous theoretical reports. ${ }^{44,51}$

Based on the electronic band structure, we calculated the intrinsic electrical thermal conductance $\boldsymbol{\sigma}_{e}$ with zero gate voltage $\left(\mu=E_{f}\right)$. Figure $5 \mathrm{a}, \mathrm{b}$ shows the armchair-direction electrical thermal conductance $\boldsymbol{\sigma}_{e \text {-armchair }}$ and zigzag-direction electrical thermal conductance $\boldsymbol{\sigma}_{e \text {-zigzag }}$ as a function of uniaxial strain at room temperature $(T=300 \mathrm{~K}) . \boldsymbol{\sigma}_{e \text {-armchair }}$ and $\boldsymbol{\sigma}_{e \text {-zigzag }}$ for strain-free $2 \mathrm{D}$ hydrogen boride are 3.56 and $2.88 \mathrm{nW} \mathrm{K}^{-1} \mathrm{~nm}^{-2}$, respectively, which are much higher than that of graphene $\left(\sim 0.3 \mathrm{nW} \mathrm{K}^{-1} \mathrm{~nm}^{-2}\right){ }^{22}$ When the strain is applied in the armchair-direction, $\boldsymbol{\sigma}_{e \text {-armchair }}$ and $\boldsymbol{\sigma}_{e \text {-zigzag }}$ increase with tensile strain. In contrast, under strain along zigzagdirection, $\boldsymbol{\sigma}_{e \text {-armchair }}$ and $\boldsymbol{\sigma}_{e \text {-zigzag }}$ decreases rapidly. The electronic conductance $G_{e}$ and electrical thermal conductance $\boldsymbol{\sigma}_{e}$ can be described by the Wiedemann-Franz law, $\sigma_{e}=L_{e} G_{e} T$, where $L_{e}$ is the Lorenz constant, $T$ is temperature. The electronic conductance $G_{e}$ is $G_{e}=n e^{2} \tau / m$, here $n$ is the carrier concentration, $\tau$ is the carrier relaxation time, and $m$ is the effective mass of charge carrier. To understand the strain effects on electrical thermal conductance, we show the electron density of states (DOS) under armchair-direction strain (Fig. 5c) and zigzag-direction strain (Fig. 5d), respectively. For strain along the armchair-direction, with tensile strain increases from 0 to $16 \%$, the metallic electronic character is completely preserved, and the DOS at the Fermi level increases from 0.78 to 1.12 , revealing higher carrier concentration induced by tensile strain, consequently leads to increase in electronic conductance and electrical thermal conductance. With armchair-strain increases from 0 to $16 \%$, $\boldsymbol{\sigma}_{e \text {-armchair }}\left(\boldsymbol{\sigma}_{e \text {-zigzag }}\right)$ increases from $3.56(2.88) \mathrm{nW} \mathrm{K}^{-1} \mathrm{~nm}^{-2}$ to 5.02 (5.18) nW K $\mathrm{nm}^{-2}$, with about $41 \%$ (79\%) increment. On the other side, the effect of zigzag-strain on the modification of the electrical thermal conductance is more pronounced, which is related to the bonding features of 2D hydrogen boride. With zigzag-strain, $\boldsymbol{\sigma}_{e \text {-armchair }}\left(\boldsymbol{\sigma}_{e \text {-zigzag }}\right)$ decreases quickly because of the reduction in density of states at Fermi level. More interesting, when strain exceeds $16 \%, \sigma_{e-\text { armchair }}\left(\boldsymbol{\sigma}_{e-\text {-zigzag }}\right)$ decreases to $0 \mathrm{nW} \mathrm{K}^{-1} \mathrm{~nm}^{-2}$, representing fully off-state of the heat channel by electron. As shown in Fig. S6, when tensile loading along zigzag-direction is applied, there is metal-to-semiconductor transition, results in significantly reduction in the DOS at the Fermi level. The band gap is $0.15 \mathrm{eV}$ at $16 \%$ tensile strain in the zigzag direction. In this material, there are three different types of bond, B-B covalent bond along the zigzag-direction, $\mathrm{B}-\mathrm{B}$ bond with hydrogen bridging along the armchair-direction, and $\mathrm{B}-\mathrm{H}$ bonds. For tensile strain along the zigzag-direction, the covalent $B-B$ bonds are oriented along the loading direction. As the electron localization occurs at the center of B-B covalent bond, it is sensitive to the loading along zigzagdirection, which results in the metal-to-semiconductor transition. ${ }^{44}$ The metal-to-semiconductor transition leads to significant reduction in the electrical thermal conductance, from about $3 \mathrm{nW} \mathrm{K}^{-1} \mathrm{~nm}^{-2}$ to 0 when tensile strain exceeds $16 \%$.

High total thermal conductance over graphene

From the phonon and electron thermal conductance, finally we explore the total thermal conductance of $2 \mathrm{D}$ hydrogen boride $\left(\sigma=\sigma_{p h}+\sigma_{e}\right)$ at room temperature, and the strain dependence. For strain-free 2D hydrogen boride, the total thermal conductance along armchair- and zigzag-direction are 7.48 and $6.96 \mathrm{nW} \mathrm{K}^{-1}$ $\mathrm{nm}^{-2}$, respectively. It is worth stressing that the thermal conductance along armchair-direction is almost two-fold higher than that of graphene, ${ }^{22,24}$ and it is the highest value among all known 2D materials. This is contributed by the similar lattice thermal conductance with respect to graphene, and the 

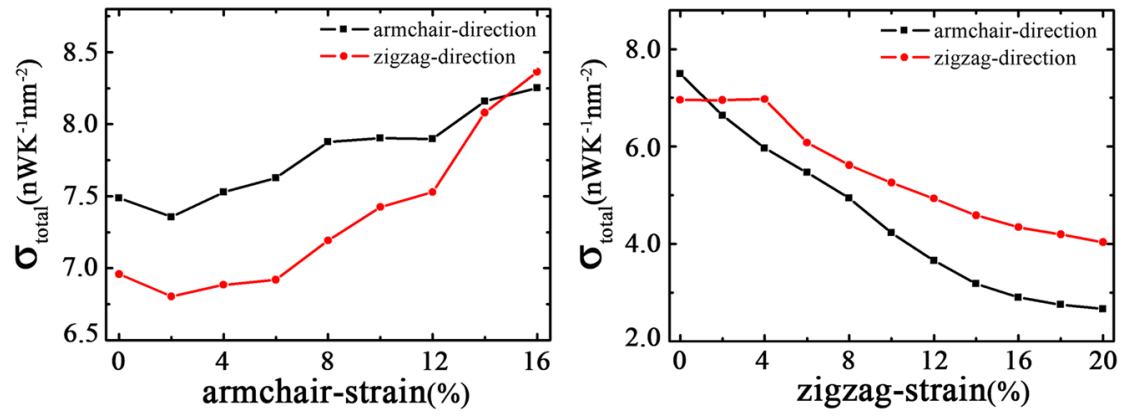

Fig. 6 The room temperature total thermal conductance as a function of strain for $\mathbf{a}$ armchair-direction strain and $\mathbf{b}$ zigzag-direction strain

significantly higher electrical thermal conductance compared with graphene.

As shown in Fig. 6, total thermal conductance of 2D hydrogen boride along both armchair- and zigzag-direction increase with armchair-strain, totally unlike the reduced phonon thermal conductance in the same range of tensile strain. In general, tensile strain will lead to reduction in thermal conductance because the reduced phonon group velocity and phonon transmission. However, for 2D hydrogen boride, abnormal tensile strain dependence occurs, because of the increased electrical thermal conductance. With armchair-strain increases from 0 to $16 \%$, phonon thermal conductance decreases from about $4 \mathrm{nW}$ $\mathrm{K}^{-1} \mathrm{~nm}^{-2}$ to about $3.2 \mathrm{nW} \mathrm{K}^{-1} \mathrm{~nm}^{-2}$. However, in the same range of tensile strain, electrical thermal conductance increases from $3 \sim 3.5 \mathrm{nW} \mathrm{K}^{-1} \mathrm{~nm}^{-2}$ to $\sim \mathrm{nW} \mathrm{K}^{-1} \mathrm{~nm}^{-2}$. The large increase in electrical thermal conductance offsets the reduction in phonon thermal conductance, and results in this abnormal strain dependence. Moreover, the effect of zigzag-strain on total thermal conductance is more remarkable. Using armchair-direction thermal conductance as an example, we can find that zigzag-strain can reduce phonon thermal conductance along armchairdirection about $40 \%$, while the reduction ratio is up to $70 \%$ for total thermal conductance. This happens because of the large contribution of electron to total thermal conductance, and the electronic bandgap opening due to zigzag-strain. Therefore, compared with other 2D materials in which phonons dominate thermal conductance, there is larger degree of freedom to modulate thermal conductance of 2D hydrogen boride.

In conclusion, we investigated phonon, mechanical and electronic properties of $2 \mathrm{D}$ hydrogen boride. Based on the firstprinciples calculation provided phonon property and electronic band structure, combined with quantum transport theory, we predicted phonon and electrical thermal conductance. The phonon thermal conductance of $2 \mathrm{D}$ hydrogen boride is close to that of graphene, while its electrical thermal conductance is almost ten times over graphene. This happens due to the high electron density of state at Fermi level. Moreover, at the Fermi level, the tensile strain along armchair-direction gives a higher electronic density of states, which effectively brings more states toward electrical thermal conductance. In contrast, zigzagdirection tensile strain induces bandgap opening at the $\Gamma$-point, which can fully "close" the electron channel, leading to zero electrical thermal conductance. This is the opposite of the armchair-direction strain effect. Our work reveals giant thermal conductance in 2D hydrogen boride, a new boron based 2D material synthesized experimentally.

\section{METHODS}

\section{DFT calculations}

In this paper, we studied electron and phonon thermal conductance of the experimentally fabricated 2D hydrogen boride sheets. The structural parameters, lattice vibrations and interatomic force constants (IFCs) are calculated from the density functional theory (DFT) by using the QuantumEspresso code, ${ }^{57}$ within the generalized gradient approximation (GGA) of the Perdew-Burke-Ernzerhof (PBE). Cutoff energies of 50 Ry for plane wave and 500 Ry for electronic density are adopted. The first Brillouin zone was sampled with a $35 \times 25 \times 1$ for the unit cell, and a vacuum layer with $15 \AA$ thickness is adopted. Each atomic structure of the $2 \mathrm{D}$ hydrogen boride was fully relaxed until the forces are less than $0.01 \mathrm{eV} / \AA$. The dynamic matrix based on a $7 \times 5 \times 1$ grid of $q$ points is calculated by density functional perturbation theory (DFPT), and giving the through inverse Fourier transform to real space.

\section{Strain effect}

For uniaxial tensile strain, we applied a series of tensile strains on the rectangular unit cell along the armchair- (or zigzag-) direction and relaxed the lattice along the zigzag- (or armchair-) direction until the corresponding conjugate stress component is less than $0.01 \mathrm{GPa}$. In the structure optimization, periodic boundary condition is applied to both armchair- and zigzag-direction, and all atoms are allowed to move. The tensile strain is defined as $\varepsilon_{i}=\left(L_{i}-L_{i 0}\right) / L_{i 0}, i=$ armchair/ zigzag. Here $L_{i}$ is the strained lattice constant along armchair- or zigzag-direction, and $L_{i 0}$ is the corresponding original lattice constant, respectively.

\section{Phonon thermal conductance}

Phonon mean free path is the key quantity for classifying transport feature. It is defined as the average transport distance of phonon between two successive scatterings arises due to the coupling between phonons and other quasi-particles, as well as phonons themselves. In physics, the phonon-phonon scattering process requires the conservation of energy and quasi-momentum. In low-dimensional nano materials, confinement effects induce energy level splitting, make the satisfaction of the conservation of energy and quasi-momentum difficult. Moreover, because of the high Debye temperature in carbon-based and boron-based materials, ${ }^{58}$ room temperature can be treated as low temperature. At room temperature, the phonon excitation is low, thus the possibility of phonon-phonon anharmonic scattering process is greatly suppressed. Consequently, carbon-based and boron-based materials usually possess long phonon mean free path. When the size of material is smaller than phonon mean free path, phonons experience almost no scatterings during transport, leading to ballistic phonon transport. In such case, thermal conductivity is not a constant but significantly depends on the system size. This phenomenon has been experimentally observed in graphene ${ }^{59}$ and carbon nanotubes. ${ }^{60}$ However, thermal conductance is well defined and does not change as the system length increases. Therefore, in this work, we focus on thermal conductance of this novel material.

The phonon thermal conductance can be obtained from the phonon non-equilibrium Green's function (NEGF) method based on the calculated force constant matrix. As shown in Fig. S1, the sample is connected to left and right heat reservoirs, which are assumed to be in thermal equilibrium with temperature $T_{H}$ and $T_{C}$, respectively. The thermal conductance can be described by the Landauer formula: ${ }^{61}$

$\sigma(T)=\frac{1}{2 \pi S} \int_{0}^{\infty} d \omega \hbar \omega \frac{\partial f(\omega)}{\partial T} \equiv(\omega)$,

where $f(\omega)$ is the Bose-Einstein distribution function for a phonon with frequency $\omega$ at the reservoirs, $S$ is the transverse cross-sectional area, $\equiv(\omega)$ 
is phonon transmission as a function of phonon frequency $\omega$, which can be evaluated according to the IFCs within the NEGF framework. ${ }^{61,62}$

The cross-section area scaled thermal conductance $\sigma$ has relationship to thermal conductivity $K$ as $\sigma=\frac{K}{L}$, where $L$ is the longitudinal length of the sample. Obviously, as thermal conductance $\sigma$ is length independent, in the ballistic regime, thermal conductivity increases linearly with system length.

Electrical thermal conductance

The electrical thermal conductance is calculated using NEGF-DFT codes in the Atomistix ToolKit (ATK) under the equilibrium conditions. ${ }^{63,64}$ The first Brillouin zone is sampled with $1 \times 100 \times 100$ Monkhorst-Pack k-mesh, and the cutoff energy is set to $150 \mathrm{Ry}$. All atomic positions are relaxed until the maximum inter-atomic force is smaller than $0.01 \mathrm{eV} / \AA$. For electron transport calculation, two semi-infinite leads are adopted as left and right electrodes, and the studied system is the central part. The electron transmission is calculated as:

$\Xi_{\mathrm{e}}[\omega]=\Xi_{\mathrm{r}}\left(\mathrm{G}^{\mathrm{r}} \Gamma_{\mathrm{L}} \mathrm{G}^{\mathrm{a}} \Gamma_{\mathrm{R}}\right)$,

where $G^{r}=\left(G^{a}\right)^{\dagger}$ is the retarded surface Green function of the central of the scattering region and $\Gamma_{L(R)}$ represents the coupling interaction with the left seim-infinite lead. Thus electronic thermal conductance $\sigma_{e}$ can be obtained by,

$\sigma_{e}=\frac{1}{T}\left[L^{(2)}-\frac{\left(L^{(1)}\right)^{2}}{L^{(0)}}\right]$

with

$L^{(n)}=\frac{2}{h} \int\left[-\frac{\partial f(E, T)}{\partial E}\right] T_{e}(E) \mathrm{dE}$,

where $f(E, T)$ is the Fermi-Dirac distribution function at temperature $T$.

\section{DATA AVAILABILITY}

The data that support the findings of this study and the code for the strategy proposed in this study are available from the corresponding author (D. F. Li) upon reasonable request.

\section{ACKNOWLEDGEMENTS}

We gratefully acknowledge funding supporting from National Natural Science Foundation of China (Grant 11604035). H.Z. and G.Z. are supported in part by a grant from the Science and Engineering Research Council (152-70-00017).

\section{AUTHOR CONTRIBUTIONS}

D.L. and G.Z. established the theoretical models and supervised the project, J.H., Y.Y., and C.F. performed the calculations and data analysis, J.H. wrote the paper, D.L. and G.Z. revised the paper. J.H., C.Z., H.Z., and P.Z. discussed the results.

\section{ADDITIONAL INFORMATION}

Supplementary information accompanies the paper on the npj Computational Materials website (https://doi.org/10.1038/s41524-019-0183-2).

Competing interests: The authors declare no competing interests.

Publisher's note: Springer Nature remains neutral with regard to jurisdictional claims in published maps and institutional affiliations.

\section{REFERENCES}

1. Fiori, G. et al. Electronics based on two-dimensional materials. Nat. Nanotech. 9, 768-779 (2014)

2. $\mathrm{Yu}$, Z. et al. Realization of room-temperature phonon-limited carrier transport in monolayer $\mathrm{MoS}_{2}$ by dielectric and carrier screening. Adv. Mater. 28, 547-552 (2016).

3. Liu, Y., Duan, X., Huang, Y. \& Duan, X. Two-dimensional transistors beyond graphene and TMDCs. Chem. Soc. Rev. 47, 6388-6409 (2018).

4. Wang, M. et al. A spectrally tunable all-graphene-based flexible field-effect lightemitting device. Nat. Commun. 6, 7767 (2015).
5. Tao, Q. et al. An intelligent artificial throat with sound-sensing ability based on laser induced graphene. Nat. Commun. 8, 14579 (2017).

6. Xie, G. et al. Ultra-low thermal conductivity of two-dimensional phononic crystals in the incoherent regime. npj Comput. Mater. 4, 21 (2018).

7. Moore, L. \& Shi, L. Emerging challenges and materials for thermal management of electronics. Mater. Today 17, 163-174 (2017).

8. Song, H. et al. Two-dimensional materials for thermal management applications. Joule 2, 442-463 (2018).

9. Balandin, A. A. Thermal properties of graphene and nanostructured carbon materials. Nat. Mater. 10, 569-581 (2011).

10. Tian, Z., Esfarjani, K. \& Chen, G. Enhancing phonon transmission across a $\mathrm{Si} / \mathrm{Ge}$ interface by atomic roughness: First-principles study with the Green's function method. Phys. Rev. B 86, 235304 (2012).

11. Zhang, G. \& Zhang, Y.-W. Thermoelectric properties of two-dimensional transition metal dichalcogenides. J. Mater. Chem. C 5, 7684-7698 (2017).

12. $\mathrm{Ma}, \mathrm{Y}$. et al. Ordered water layers by interfacial charge decoration leading to an ultra-low Kapitza resistance between graphene and water. Carbon N. Y. 135, 263-269 (2018)

13. Sadeghi, M., Pettes, M. \& Shi, L. Thermal transport in graphene. Solid State Comm. 152, 1321-1330 (2012).

14. Yang, N., Xu, X., Zhang, G. \& Li, B. Thermal transport in nanostructures. AIP Adv. 2 , 041410 (2012).

15. Dubi, Y. \& Ventra, M. Colloquium: Heat flow and thermoelectricity in atomic and molecular junctions. Rev. Mod. Phys. 83, 131 (2011).

16. Marconnet, M., Panzer, A. \& Goodson, E. Thermal conduction phenomena in carbon nanotubes and related nanostructured materials. Rev. Mod. Phys. 85, 1295 (2013).

17. Zhang, G. \& Zhang, Y.-W. Thermal properties of two-dimensional materials. Chin. Phys. B 26, 034401 (2017).

18. Kou, L., Chen, C. \& Smith, S. C. Phosphorene: fabrication, properties, and applications. J. Phys. Chem. Lett. 6, 2794-2805 (2015).

19. Hong, Y., Zhang, J. X. \& Zeng, X. Thermal transport in phosphorene and phosphorene-based materials: A review on numerical studies. Chin. Phys. B 27, 036501 (2018).

20. Cahill, G. et al. Nanoscale thermal transport. II. 2003-2012. Appl. Phys. Rev. 1, 011305 (2014).

21. Mu, M., Wan, C. \& Mcnally, T. Thermal conductivity of $2 \mathrm{D}$ nano-structured graphitic materials and their composites with epoxy resins. 2D materials 4, 042001 (2017).

22. Zhou, H., Cai, Y., Zhang, G. \& Zhang, Y.-W. Quantum thermal transport in stanene Phys. Rev. B 94, 045423 (2016).

23. Guo, Z.-X., Zhang, D. \& Gong, X.-G. Manipulating thermal conductivity through substrate coupling. Phys. Rev. B 84, 075470 (2011).

24. Xu, Y., Chen, X., Gu, B.-L. \& Duan, W. Intrinsic anisotropy of thermal conductance in graphene nanoribbons. Appl. Phys. Lett. 95, 233116 (2009).

25. Wang, Y., Yang, C., Cheng, Y. \& Zhang, Y. A molecular dynamics study on thermal and mechanical properties of graphene-paraffin nanocomposites. RSC Advances 5, 82638-82644 (2015).

26. Xie, Z., Chen, K. \& Duan, W. Thermal transport by phonons in zigzag graphene nanoribbons with structural defects. J. Phys. Condens. Matter. 23, 315302 (2011).

27. Nishino, $\mathrm{H}$. et al. Formation and characterization of hydrogen boride sheets derived from $\mathrm{MgB}_{2}$ by cation exchange. J. Am. Chem. Soc. 139, 13761-13769 (2017)

28. Penev, E. S., Kutana, A. \& Yakobson, B. I. Can two-dimensional boron super conduct? Nano Lett. 16, 2522-2526 (2016).

29. Zhang, Z., Yang, Y., Gao, G. \& Yakobson, B. I. Two-dimensional boron monolayers mediated by metal substrates. Angew. Chem. Int. Edit. 54, 13022-13026 (2015).

30. Liu, Y., Penev, E. S. \& Yakobson, B. I. Probing the synthesis of two-dimensional boron by first-principles computations. Angew. Chem. Int. Edit. 52, 3156-3241 (2013).

31. Liu, H., Gao, J. \& Zhao, J. From boron cluster to two-dimensional boron sheet on $\mathrm{Cu}$ (111) surface: growth mechanism and hole formation. Sci. Rep. 3, 3238 (2013).

32. $\mathrm{Wu}, \mathrm{X}$. et al. Two-dimensional boron monolayer sheets. ACS Nano 6, 7443-7453 (2012).

33. Mannix, J. et al. Synthesis of borophenes: anisotropic, two-dimensional boron polymorphs. Science 350, 1513-1516 (2015)

34. Feng, B. et al. Experimental realization of two-dimensional boron sheets. Nat. Chem. 8, 563 (2016).

35. Rao, D. et al. Ultrahigh energy storage and ultrafast ion diffusion in borophenebased anodes for rechargeable metal ion batteries. J. Mater. Chem. A. 5, 2328-2338 (2017).

36. Kulish, V. V. Surface reactivity and vacancy defects in single-layer borophene polymorphs. Phys. Chem. Chem. Phys. 19, 11273-11281 (2017).

37. Xiang, P. et al. Metallic borophene polytypes as lightweight anode materials for non-lithium-ion batteries. Phys. Chem. Chem. Phys. 19, 24945-24954 (2017). 
38. Jena, N., Araujo, R., Shukla, V. \& Ahuja, R. Borophane as a benchmate of graphene: apotential 2D material for anode of $\mathrm{Li}$ and $\mathrm{Na}$-ion batteries. ACS Appl. Mater. Inter. 9, 16148-16158 (2017).

39. Shukla, V., Grigoriev, A., Jena, N. \& Ahuja, R. Strain controlled electronic and transport anisotropies in two-dimensional borophene sheets. Phys. Chem. Chem. Phys. 20, 22952-22960 (2018).

40. Shukla, V., Grigoriev, A., Jena, N. \& Ahuja, R. Borophene's tryst with stability: exploring 2D hydrogen boride as an electrode for rechargeable batteries. Phys. Chem. Chem. Phys. 20, 22008-22016 (2018).

41. Chen, L. et al. Reversible hydrogen storage in pristine and Li decorated 2D boron hydride. Phys. Chem. Chem. Phys. 20, 30304-30311 (2018).

42. Makaremi, M., Mortazavi, B. \& Singh, C. 2D hydrogenated graphene-like borophene as a high capacity anode material for improved Li/Na batteries: A first principles study. Mater. Today Energy 8, 22-28 (2018).

43. Lee, $C$. et al. Peculiar bonding associated with atomic doping and hidden honeycombs in borophene. Phys. Rev. B 97, 075430 (2018).

44. Mortazavi, B. et al. Borophene hydride: a stiff 2D material with high thermal conductivity and attractive optical and electronic properties. Nanoscale 10, 3759-3768 (2018).

45. Zhou, H., Cai, Y., Zhang, G. \& Zhang, Y.-W. Superior lattice thermal conductance of single layer borophene. npj 2D Mater. Appl. 1, 14 (2017).

46. Li, D. et al. Stretch-driven increase in ultrahigh thermal conductance of hydrogenated borophene and dimensionality crossover in phonon transmission. Adv. Funct. Mater. 28, 1801685 (2018).

47. Li, D. et al. Review of thermal transport and electronic properties of borophene. Chin. Phys. B 27, 036303 (2018).

48. Zhang, Z., Yang, Y., Penev, E. \& Yakobson, B. I. Elasticity, flexibility, and ideal strength of borophenes. Adv. Funct. Mater. 27, 1605059 (2017).

49. Cheng, $C$. et al. Suppressed superconductivity in substrate-supported $\beta_{12}$ borophene by tensile strain and electron doping. 2D Mater. 4, 025032 (2017).

50. Mogulkoc, A., Mogulkoc, Y., Kecil, K. \& Durgun, E. the effect of strain and functionalization on the optical properties of borophene. Phys. Chem. Chem. Phys. 20, 21043-21050 (2018).

51. Jiao, Y., Ma, F., Bell, J., Bilic, A. \& Du, A. Two-dimensional boron hydride sheets: high stability, massless dirac fermions, and excellent mechanical properties. Angew. Chem. Int. Edit. 128, 10448-10451 (2016).

52. Wu, W. et al. Giant mechano-optoelectronic effect in an atomically thin semiconductor. Nano Lett. 18, 2351-2357 (2018).

53. Conley, H.-J. et al. Bandgap engineering of strained monolayer and bilayer $\mathrm{MoS}_{2}$. Nano Lett. 13, 3626-3630 (2013).
54. Zhang, G. \& Zhang, Y.-W. Strain effects on thermoelectric properties of twodimensional materials. Mech. Mater. 91, 382-398 (2015).

55. Li, Y. et al. Giant anisotropic raman response of encapsulated ultrathin black phosphorus by uniaxial strain. Adv. Funct. Mater. 27, 1600986 (2017).

56. Wang, Z., Lu, T. Y., Wang, H. Q., Feng, Y. P. \& Zheng, J. C. High anisotropy of fully hydrogenated borophene. Phys. Chem. Chem. Phys. 18, 31424-31430 (2016).

57. Giannozzi, P. et al. QUANTUM ESPRESSO: a modular and open-source software project for quantum simulations of materials. J. Phys. Condens. Matter. 21, 395502 (2009).

58. Zhang, Z., Penev, E.-S. \& Yakobson, B. I. Two-dimensional boron: structures, properties and applications. Chem. Soc. Rev. 46, 6746-6763 (2017).

59. $\mathrm{Xu}, \mathrm{X}$. et al. Length-dependent thermal conductivity in suspended single-layer graphene. Nat. Commun. 5, 3689 (2014).

60. Lee, V., Wu, C.-H., Lou, Z., Lee, W. \& Chang, C. Divergent and Ultrahigh thermal conductivity in millimeter-long nanotubes. Phys. Rev. Lett. 118, 135901 (2017).

61. Wang, J.-S., Wang, J. \& Lü, J. T. Quantum thermal transport in nanostructures. Eur. Phys. J. B 62, 381-404 (2008).

62. Zhang, W., Fisher, T. S. \& Mingo, N.Simulation of interfacial phonon transport in $\mathrm{Si}-\mathrm{Ge}$ heterostructures using an atomistic Green's function method. ASMEJ. Heat Transfer. 129, 483-491 (2007).

63. Taylor, J., Guo, H. \& Wang, J. Ab initio modeling of quantum transport properties of molecular electronic devices. Phys. Rev. B 63, 245407 (2001).

64. Brandbyge, M., Mozos, J., Ordejon, P., Taylor, J. \& Stokbro, K. Density-functional method for nonequilibrium electron transport. Phys. Rev. B 65, 165401 (2002).

(C) Open Access This article is licensed under a Creative Commons Attribution 4.0 International License, which permits use, sharing, adaptation, distribution and reproduction in any medium or format, as long as you give appropriate credit to the original author(s) and the source, provide a link to the Creative Commons license, and indicate if changes were made. The images or other third party material in this article are included in the article's Creative Commons license, unless indicated otherwise in a credit line to the material. If material is not included in the article's Creative Commons license and your intended use is not permitted by statutory regulation or exceeds the permitted use, you will need to obtain permission directly from the copyright holder. To view a copy of this license, visit http://creativecommons. org/licenses/by/4.0/.

(c) The Author(s) 2019 